\title{
Two collaborative experiments in star cluster evolution
}

\author{
Douglas C. Heggie \\ University of Edinburgh, Department of Mathematics and Statistics, \\ King's Buildings, Edinburgh EH9 3JZ, UK
}

\begin{abstract}
We summarise the specification and some previously unpublished results of the collaborative experiment ("Kyoto I") that was carried out at the time of the Kyoto General Assembly of the IAU. As the subject has advanced considerably since then, and codes have become more elaborate, we describe an agreed proposal for a new experiment ("Kyoto II").
\end{abstract}

\section{Introduction}

Results from the very first collaborative experiment, with $N=25$ stars, were actually presented, not in 1997 in Kyoto, but thirty years earlier at a meeting in Paris in 1967 (Lecar 1968). Calculations for the Kyoto experiment (which will here be referred to as Kyoto I) took place mainly in the run-up to the Kyoto General Assembly of the IAU in 1997, where the results were presented. It involved not only $N$-body integrations (up to $N=65536$ !) but also various kinds of Fokker-Planck and even gaseous models (Heggie et al 1998). Some hitherto unpublished results are presented in the next section, along with a summary of its specification.

Kyoto I was restricted to pure stellar dynamics without primordial binaries. As the subject extends into the realm of "all-inclusive", more realistic simulations, including the presence of primordial binaries and the simulation of stellar evolution, it is timely to initiate a more advanced collaboration. In section 3 we present and discuss the specification of a new problem which was largely agreed during IAU Symposium 208 in Tokyo, though it will be referred to as Kyoto II. Computations are only just beginning at the time of writing, and results will be presented elsewhere.

\section{2. "First" collaborative experiment (Kyoto I)}

During the run-up to the IAU General Assembly in Kyoto, i.e. the period MayAugust 1997, several groups around the world participated in a collaborative experiment in star cluster evolution. The aim was to study the evolution of the same cluster by several different techniques. The initial specification of the cluster was as follows:

1. King Model, $W_{0}=3$ (non-rotating), $r_{t}=30 \mathrm{pc}, M=6 \times 10^{4} M_{\odot}$;

2. mass function $n(m) d m \propto m^{-2.35} d m$ for $0.1 M_{\odot}<m<1.5 M_{\odot}$; 


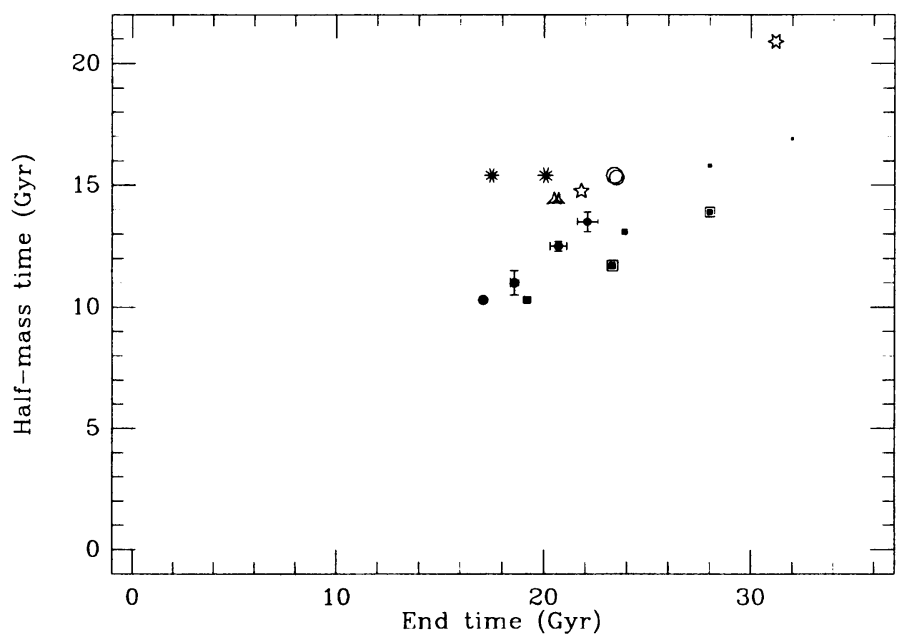

Figure 1. "First" collaborative experiment: time at which half of the mass had escaped, against end time. The origin is included to give an impression of the relative variation in the results. Some $N$-body computations include data from several realisations of the specification, and then error bars are given. In general, the size of the symbol relates to some aspect of the reliability of the model, e.g. the number of mass bins used in a Fokker-Planck simulation. In some cases, especially with the Monte Carlo code, a simulation can continue for a lengthy period with almost negligible mass, and the end time gives a poor indication of its reliability. The fastest evolving $N$-body models are those with largest $N$.

3. No (primordial) binaries, no mass segregation;

4. Tidal boundary conditions corresponding to circular motion round a pointmass galaxy;

5. Heating by 3 -body binaries;

6. No stellar evolution, no collisions.

The 1997 collaborative experiment brought a number of benefits. In particular, new stellar dynamics was learned, because of problems raised by the $N$-body results (see Baumgardt 2001), and the specification has become a kind of benchmark problem which has been occasionally used since (e.g. Giersz 2001). Though only one publication has emerged so far, a fault for which the author bears total responsibility, considerable amounts of data became available almost immediately on the web (http://www.maths.ed.ac.uk/ douglas/experiment.html). One drawback of the specification also emerged. Data had been requested corresponding to times when the system had lost half of its mass (by escape) and at the time of core collapse. Unfortunately these times turned out to be rather similar. 


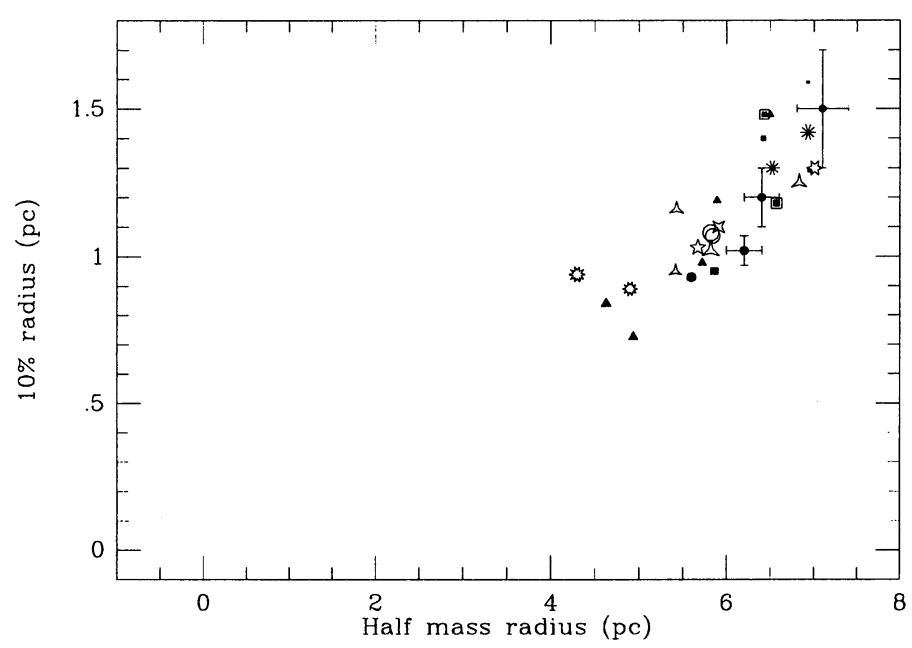

Figure 2. "First" collaborative experiment. Half-mass and $10 \%$ Lagrangian radii at the time of core bounce.

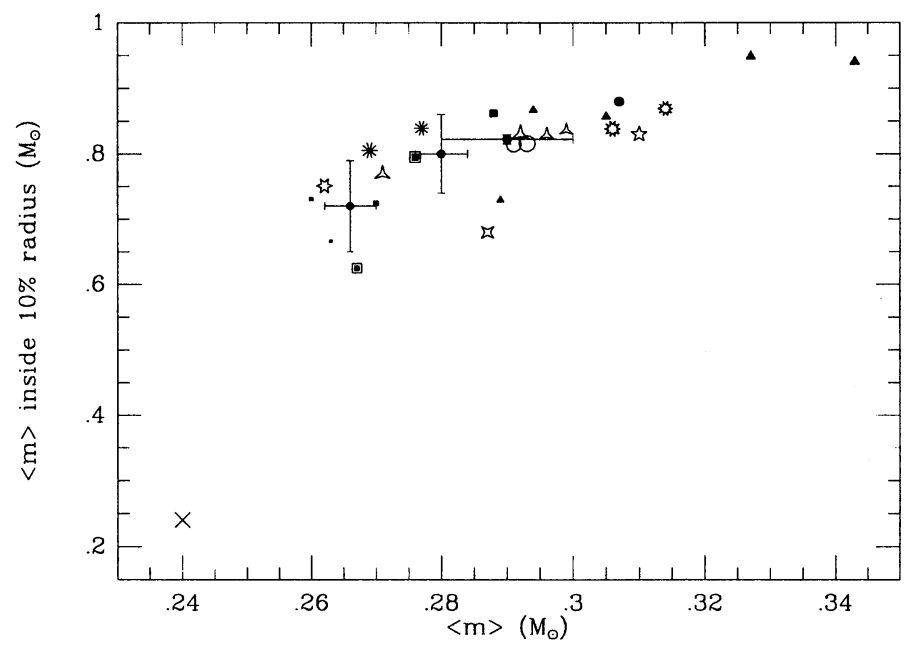

Figure 3. "First" collaborative experiment. Global mean stellar mass, and mean mass within the $10 \%$ Lagrangian radius, at the time of core bounce. The cross at lower left gives the initial values. For a power law mass function, the spread in abscissa values at core bounce corresponds to a variation in the power law index from about 2.2 to 1.7 ; for the ordinates the range is from about -0.7 to +0.7 . 


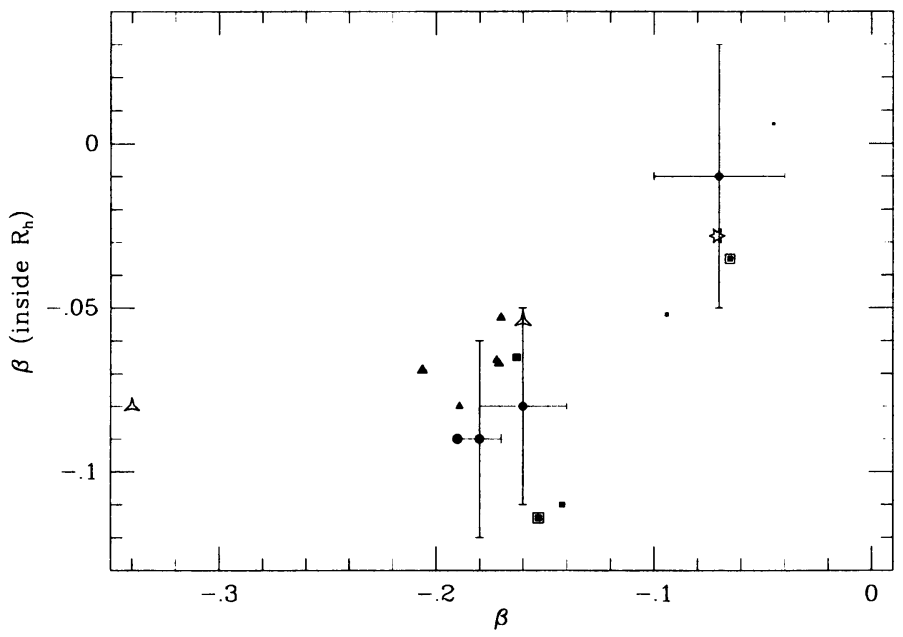

Figure 4. "First" collaborative experiment. Global anisotropy $\beta$, and anisotropy within the half-mass radius, at the time of core bounce. $\beta$ is defined to be $1-\left\langle v_{t}^{2}\right\rangle /\left(2\left\langle v_{r}^{2}\right\rangle\right)$, where $v_{r}$ and $v_{t}$ are radial and transverse velocities, and the averages are not mass weighted.

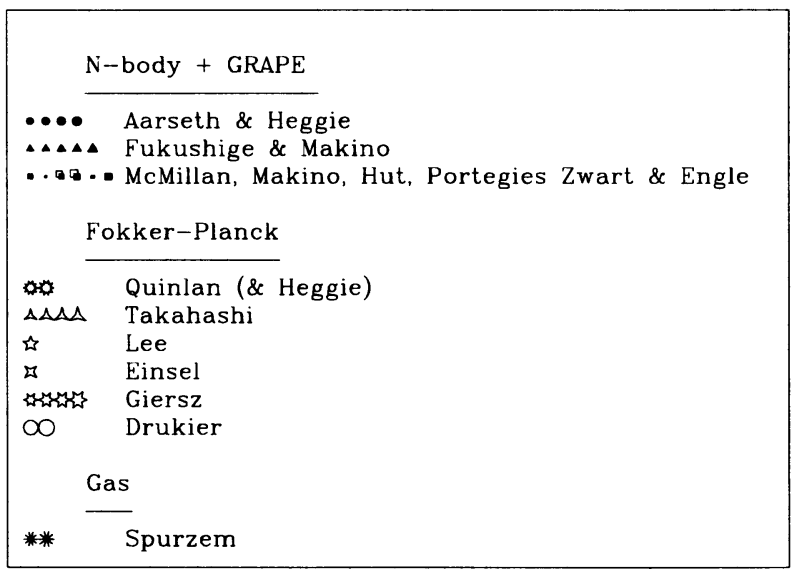

Figure 5. "First" collaborative experiment. Key to the preceding figures. 
Figs 1-4 illustrate some of the data which emerged from this experiment. Other data can be found in Heggie et al 1998. The key to the symbols is given in Fig.5, which also lists the persons who participated. In studying these figures it must be recalled that some improvements have been made in these codes since the time of the collaborative experiment, most notably in the treatment of boundary conditions in Fokker-Planck codes (Takahashi \& Portegies Zwart 1998, 2000).

\section{A new proposal}

\subsection{Specification}

Now that $N$-body simulations in stellar dynamics are attempting a new level of realism, it is timely to build on the previous collaborative experiment by devising a more ambitious specification. The following specification had been under discussion for some time before the time of the symposium, and was largely firmed up then. The aim has been to devise a model which displays an interesting interplay of stellar and dynamical evolution, and is doable by as many groups as possible. The simulation of the recent evolution of M67 by Hurley et al (2001) has been a very useful guide. Their model, with $N_{s}=5000$ single stars and $N_{b}=5000$ binaries (i.e. $50 \%$ primordial binaries and $N=15000$ stars altogether) took about 1 month on a GRAPE 4.

The agreed specification is as follows:

- Initial structure

1. King (1966) model, $W_{0}=5$

2. no initial mass segregation

- Boundary condition

3. tidal radius $r_{t}=27.9 \mathrm{pc}$ (initially)

4. tidal cutoff (i.e. instantaneous removal at $r_{t}$ )

- Single stars

5. $N_{s}=12288$

6. IMF: KTG3 (Kroupa, Tout \& Gilmore $1993^{1}$ ), restricted to $0.1 M_{\odot}<$ $m<100 M_{\odot}$

- Primordial binaries

7. $N_{b}=4096$

8. eccentricity distribution $f(e)=2 e$

9. IMF for total binary mass: KTG1 (see Hurley et al 2001, eq.(1)), restricted to $0.2 M_{\odot}<m<200 M_{\odot}$

\footnotetext{
${ }^{1}$ To be precise, their eq.(14), using entries in Table 10 with $\alpha_{1}=1.3$
} 
10. mass ratio $q<1$, uniform in the range giving component masses in the range $0.1 M_{\odot}<m<100 M_{\odot}$

11. $a$ : uniform in $\log a$ in a range such that $a(1-e)>2\left(R_{1}+R_{2}\right)$ and binding energy exceeds $10 k T^{2}$

- Stellar evolution

12. $Z=$ solar

\subsection{Discussion}

Initial conditions, suitable for $N$-body computations, have been constructed in accordance with the above specification ${ }^{3}$. It was decided to do this once for all, and not to invite each participant to create his own set of initial conditions. The reason for this is that different initial masses will lead directly to binaries with very different histories, whereas use of a common set of initial conditions may allow the fate of the same binary in different simulations to be compared. The distributions of $q$ and $\ln a$ in the initial conditions are not, of course, uniform, as the ranges of $q$ and $a$ depend, respectively, on the total mass of a binary and the masses and radii of the components.

It follows from the above specification that the initial proportion of primordial binaries is $f_{b}=25 \%$, and that the total initial number of stars is $N=N_{s}+2 N_{b}=20480$. The initial conditions which have been constructed lead to a total initial mass $M=10174 M_{\odot}$ approximately. For a point-mass galaxy, such a mass yields the required tidal radius at a galactocentric distance of $8.5 \mathrm{kpc}$ if the circular speed is $220 \mathrm{~km} / \mathrm{s}$.

Fig 6 provides a summary of the kinds of dynamical and other processes which the two collaborative experiments involve. It also gives an indication of the kinds of computer code to which the specified problems are accessible. Even the new proposed experiment does not test certain aspects of the dynamics: unsteady tides, for example (as for a cluster on an elliptical galactic orbit, or with disk shocking), or rotation (cf. Kim et al 2001).

Notice the choice of a tidal cutoff, rather than the more realistic tidal field adopted in the "first" collaborative experiment. The reason for this is that it has become clear that implementation of tidal boundary conditions in the FokkerPlanck method is a delicate issue, and it is in the context of a tidal cutoff that the problems have been most thoroughly studied (Takahashi \& Portegies Zwart 1998, 2000).

In much the same way, the value of $N$ here is much less relevant to the dynamical evolution of globular clusters than the value selected in the "first" experiment. One reason is that it is known to be difficult to scale $N$-body results to large enough $N$, though the problems are minimised by the use of a tidal cutoff (Baumgardt 2001). In principle, the collision cross section can be scaled (Portegies Zwart et al 1999) but, more importantly, there is no experience in the scaling (with $N$ ) of $N$-body models which include stellar evolution.

\footnotetext{
${ }^{2}$ Stellar radii $R_{i}$ are from Eggleton, Fitchett \& Tout 1989, and $1.5 k T$ is the mean kinetic energy of single stars and the barycentres of binaries
}

${ }^{3}$ See the web page http://www.maths.ed.ac.uk/ douglas/kyotoII.html 
Kyoto I

\begin{tabular}{|l|l|l|l|}
\hline & Gas & Fokker-Planck & N-body \\
\hline Anisotropy & & & \\
\hline $\begin{array}{l}\text { Two-body } \\
\text { relaxation }\end{array}$ & & & \\
\hline $\begin{array}{l}\text { 3-body binary } \\
\text { heating }\end{array}$ & & & \\
\hline Steady tide & & & \\
\hline Large N & & & \\
\hline
\end{tabular}

Key

\begin{tabular}{|l|l|}
\hline Available now & \\
\hline Possible now & \\
\hline Problematic now & \\
\hline
\end{tabular}

Kyoto II

\begin{tabular}{|l|l|l|l|}
\hline & Gas & Fokker-Planck & N-body \\
\hline Anisotropy & & & \\
\hline $\begin{array}{l}\text { Two-body } \\
\text { relaxation }\end{array}$ & & & \\
\hline $\begin{array}{l}\text { 3-body binary } \\
\text { heating }\end{array}$ & & & \\
\hline Tidal cutoff & & & \\
\hline Modest N & & & \\
\hline $\begin{array}{l}\text { Primordial } \\
\text { binaries }\end{array}$ & & & \\
\hline $\begin{array}{l}\text { Single-Star } \\
\text { Evolution }\end{array}$ & & & \\
\hline Collisions & & & \\
\hline BSE* & & & \\
\hline
\end{tabular}

*Binary star evolution

Figure 6. The dynamical and other features of the two collaborative experiments. "Available now" means that the author is aware of the existence of at least one version of this kind of code where the stated feature is implemented. "Possible now" means that it could be implemented within the time scale of the current experiment, but not that this would necessarily be straightforward or easy. "Problematic now" means that there are unsolved difficulties. 


\subsection{Output}

According to results of Vesperini \& Heggie (1997) and Portegies Zwart et al (2001b) (see Heggie, this vol.), if binaries were treated as single stars with the combined mass of both components, and if stellar evolution were neglected, the lifetime in a tidal field would be 4.0Gyr. Nevertheless, an unconfirmed computation with "inert" binaries (see below) implies that the lifetime within a tidal cutoff is about $2 \mathrm{Gyr}$. Therefore an output interval of $1 \mathrm{Gyr}$ would be too small. In fact the specification of the collaborative experiment requires output at intervals of $0.5 \mathrm{Gyr}$ (at least), and the minimal required output is

1. Mass $M$

2. Half-mass radius $r_{h}$

3. $N_{s}, N_{b}$

4. Number of blue stragglers, $N_{b s}$, and degenerate stars, $N_{d e g}$

5. Luminosity $L$

Optional additional output includes

1. Luminosity function

2. Mass function

3. Colour-magnitude diagram

and no doubt more.

\subsection{Partial calculations}

The issue of stellar evolution is left largely unspecified in the above list, except for the metallicity, $Z$. Many codes which might be used for the study of the evolution of globular clusters lack all but the most rudimentary treatment of stellar evolution. Appropriate sources of formulae on single star evolution are Eggleton, Fitchett \& Tout (1989) and Hurley, Pols \& Tout (2000). Evolution of binary stars is even more involved, but suitable treatments are described in Tout, Aarseth \& Pols (1997) and Portegies Zwart et al (2001a), and in the references in the latter.

In principal, stellar evolution, where it is lacking, could be implemented in several codes within the time scale of the collaborative experiment. Even so, it is very likely that the number of participants in the full calculation will be very small. But there is also a set of restricted but useful computations for which results could be achieved by many more groups. These are

1. Stellar evolution mass loss only This approach, adopted by Chernoff \& Weinberg (1990), may be updated through use of the formulae of Eggleton et al (1989) for the evolution time. At this time, the mass of each star is replaced by the appropriate remnant mass.

2. No stellar evolution In this case the evolution is restricted to pure dynamical evolution, as in the "first" collaborative experiment, though now including primordial binaries. 
3. Inert binaries Here, even the internal degrees of freedom of the binaries are ignored. Suitable $N$-body initial conditions are also available from the web page.

\subsection{Timetable}

The idea of a second collaborative experiment arose in the spring of 2001, and Table 1 summarises the time scale to which the collaborative experiment should work.

Table 1. Timetable of collaborative experiment

\begin{aligned} & Until end August 2001 Discussion of specification \\ & Until end February 2002 "Blind" calculations (see text) \\ & February 2002 Publication of data, followed by revised calculations \\ & June 2002 First discussion of results \\ & \hline\end{aligned}

During the period when "blind" calculations are carried out, the only results which will be made available are a specification of the kind of calculation that has been carried out (e.g. "inert binaries, Fokker-Planck"). At the end of February, 2002, the results obtained so far will be made available on the web page. This may reveal shortcomings or disagreements in the calculations which have been made, and so there will be an opportunity for revised calculations to be performed before the results are summarised publicly. This initial public account of the results may be given at a workshop on the topic Integrating Stellar Evolution and Stellar Dynamics, June 17-21, 2002, at the American Museum of Natural History, New York City. In due course a definitive paper will be prepared, discussing the results in detail.

Acknowledgments. I thank the organisers for their support, and Piet Hut, who originally suggested the idea of a second collaborative experiment. I am grateful to colleagues from the first experiment, S.J. Aarseth, G. Drukier, T. Fukushige, M. Giersz, H.M. Lee, R. Spurzem and K. Takahashi for permission to include a summary of the results in this paper. I apologise to other participants whom I was unable to contact before the deadline for submission.

\section{References}

Baumgardt, H. 2001, MNRAS, 325, 1323

Chernoff, D. F. \& Weinberg, M. D. 1990, ApJ, 351, 121

Eggleton, P.P, Fitchett, M.J., Tout, C.A. 1989, ApJ, 347, 998

Giersz, M. 2001, MNRAS, 324, 218

Heggie, D. C., Giersz, M., Spurzem, R., \& Takahashi, K. 1998, Highlights in Astronomy, 11, 591

Hurley, J. R., Pols, O. R., \& Tout, C. A. 2000, MNRAS, 315, 543

Hurley, J. R., Tout, C. A., Aarseth, S. J., \& Pols, O. R. 2001, MNRAS, 323, 630 
Kim, E., Einsel, C., Lee, H.M., Spurzem, R., Lee, M.G. 2001, MNRAS, submitted; astro-ph/0109062

King, I. R. 1966, AJ, 71, 64

Kroupa, P., Tout, C. A., \& Gilmore, G. 1993, MNRAS, 262, 545

Lecar, M. 1968, Bull. Astron., 3, 91

Portegies Zwart, S.F., Makino, J., McMillan, S.L.W., Hut, P. 1999, A\&A, 348, 117

Portegies Zwart, S.F., McMillan, S.L.W., Hut, P., Makino, J. 2001a, MNRAS, 321,199

Portegies Zwart, S.F., Makino, J., McMillan, S.L.W., Hut, P. 2001b, ApJ, 546L, 101

Takahashi, K. \& Portegies Zwart, S. F. 1998, ApJ, 503, L49

Takahashi, K. \& Portegies Zwart, S. F. 2000, ApJ, 535, 759

Tout, C.A., Aarseth, S.J., Pols, O. 1997, MNRAS, 291, 732

Vesperini, E. \& Heggie, D. C. 1997, MNRAS, 289, 898 